\title{
Electrochemical potentials of layered oxide and olivine phosphate with aluminum substitution: A first principles study
}

\author{
ARUN KUMAR VARANASI, PHANI KANTH SANAGAVARAPU, ARGHYA BHOWMIK, \\ MRIDULA DIXIT BHARADWAJ*, BALASUBRAMANIAN NARAYANA, UMESH V WAGHMARE ${ }^{\dagger}$, \\ DIPTI DEODHARE ${ }^{\dagger \dagger}$ and ALIND SHARMA ${ }^{\dagger \dagger}$ \\ Centre for Study of Science, Technology and Policy, Dr Raja Ramanna complex, Bangalore 560 001, India \\ $\dagger$ Jawaharlal Nehru Centre for Advanced Scientific Research, Jakkur, Bangalore 560 064, India \\ ${ }^{\dagger}$ Centre for Artificial Intelligence and Robotics, DRDO Complex, C.V. Raman Nagar, Bangalore 560093 , India
}

MS received 26 July 2012; revised 17 September 2012

\begin{abstract}
First-principles prediction of enhancement in the electrochemical potential of $\mathrm{LiCoO}_{2}$ with aluminum substitution has been realized through earlier experiments. For safer and less expensive Li-ion batteries, it is desirable to have a similar enhancement for alternative cathode materials, $\mathrm{LiFePO}_{4}$ and $\mathrm{LiCoPO}_{4}$. Here, we present first-principles density functional theory based analysis of the effects of aluminum substitution on electrochemical potential of $\mathrm{LiCoO}_{2}, \mathrm{LiFePO}_{4}$ and $\mathrm{LiCoPO}_{4}$. While Al substitution for transition metal results in increase in electrochemical potential of $\mathrm{LiCoO}_{2}$, it leads to reduction in $\mathrm{LiFePO}_{4}$ and $\mathrm{LiCoPO}_{4}$. Through comparative topological analysis of charge density of these materials, we identify a ratio of Bader charges that correlates with electrochemical potential and determine the chemical origin of these contrasting effects: while electronic charge from lithium is transferred largely to oxygen in $\mathrm{LiCoO}_{2}$, it gets shared by the oxygen and $\mathrm{Co} / \mathrm{Fe}$ in olivine phosphates due to strong covalency between $\mathrm{O}$ and $\mathrm{Co} / \mathrm{Fe}$. Our work shows that covalency of transition metal-oxygen bond plays a key role in determining battery potential.
\end{abstract}

Keywords. Lithium ion battery; cathodes; density functional theory; density of states; Bader charge analysis; electrochemical potential.

\section{Introduction}

Electrochemical systems such as batteries, fuel cells, solar cells and super capacitors are becoming increasingly important due to their wide applicability in sustainable energy systems and storage applications. There is a wide scope for improving these systems further with the use of new materials and better designs. Electrochemical properties such as voltage, ionic diffusion coefficient, phase stability and charge storing capacity have to be significantly improved to make these systems readily available for various futuristic applications. These properties are related to other important properties like specific energy, specific power and life span of battery. The cost of the battery for a given performance is also important and depends on the abundance of key materials, routes to synthesis and fabrication techniques.

Layered oxides such as $\mathrm{LiCoO}_{2}, \mathrm{LiAl}_{x} \mathrm{Ni}_{y} \mathrm{Co}_{1-x-y} \mathrm{O}_{2}$, $\mathrm{LiNiO}_{2}$ and their derivatives have been commonly used in batteries (Ebner et al 1994; Sathiya et al 2011; Shalini et al 2001). Though the electrochemical potentials of these materials are attractive, the batteries based on them are expensive due to high cost of cobalt and nickel. $\mathrm{LiFePO}_{4}$ with an

\footnotetext{
*Author for correspondence (mdixit@ cstep.in)
}

olivine structure has been identified as a good battery cathode material (Padhi et al 1997). It is cost effective, but it has low electrochemical potential compared to cathode materials based on $\mathrm{LiCoO}_{2}$. Hence, it is important to find ways to increase the battery voltage of iron based olivine phosphates.

The development of advanced materials for batteries can be accelerated by using computational methods. Quantum mechanics, which deals with matter at electronic level, is fundamental in understanding materials properties and gives us detailed explanation regarding proper choice of materials for specific applications. First-principles density functional theory (DFT) simulations are computationally demanding but are reasonably accurate and have been successful in predicting properties of battery cathode materials (Ceder et al 1997, 1998; Kang et al 2006; Ong et al 2008). Computational screening of materials speeds up the process of discovery by saving on cost and time. Studying different classes of cathode materials ideally will give understanding of effects of various parameters such as transition metal, anion groups and structure on relevant electrochemical properties. Thus we can form universal guide lines which enable prediction of new materials. Ceder $e t$ al have been successful in designing cathode materials using this approach (Ceder et al 1998; Kang et al 2006; Ong et al 2008; Zhou et al 2004a).

Previous first principle studies as well as experiments have shown that aluminum substitution in $\mathrm{LiCoO}_{2}$ increases the 
potential (Ceder et al 1998). This has been attributed to higher oxygen participation in the electron exchange process. There is a need for quantitative understanding of this behaviour. Though theoretical investigations of trivalent substitution (such as $\mathrm{Al}$ ) in place of transition metal in olivine phosphates are reported by Wang et al (2012), the effect on the electrochemical potential with substitution is not investigated. In this work, we confirm the effect of aluminum substitution on properties of $\mathrm{LiCoO}_{2}$ and obtain new results on $\mathrm{Li}(\mathrm{Fe} / \mathrm{Co}) \mathrm{PO}_{4}$. As substitutions at higher concentration can be simulated with a system with fewer number of atoms (hence, lower computational cost), simulations at higher concentration are typically used to learn about effects of substitution at lower concentrations in many contexts. We analyse all these results by using the powerful tool of Bader's (1990) topological analysis of charge density to estimate quantitatively the charge transfer occurring in the process.

\section{Methodology}

First-principles density functional theory based simulations of lithium cobalt oxide, lithium cobalt phosphate and lithium iron phosphate with varying aluminum substitutions are carried out using Projector Augmented Wave (PAW) (Blochl 1994; Kresse and Joubert 1999) method as implemented in Vienna ab initio Simulation Package (VASP). We use a generalized gradient approximation (GGA) of Perdew-Wang type (PW91) (Perdew et al 1992) for the exchange correlation energy functionals. The initial structures used for structural optimization were either taken from inorganic crystal structure database (ICSD) or constructed in the space groups, i.e. $R \overline{3} m$ or Pnma. We developed an automation script to do the convergence calculations for relaxation to get the appropriate energy cutoff on plane wave basis and also $k$-mesh grid in sampling Brillouin-zone integration. This program starts with the initial $k$-mesh grid depending on the unit cell lattice parameters and $k$-mesh grid is increased until the ground state energy converges within $0.001 \mathrm{eV} /$ atom. With the optimized $k$-mesh grid, the energy cutoff is also chosen to satisfy the above criterion. We found $920 \mathrm{eV}$ as energy cutoff for both oxide and phosphate materials and $k$-mesh grids of $8 \times 8 \times 1,3 \times 4 \times 4$ and $3 \times 4 \times 4$, respectively for $\mathrm{LiCoO}_{2}, \mathrm{LiCoPO}_{4}$ and $\mathrm{LiFePO}_{4}$ ensured convergence. The ground states of $\mathrm{LiFePO}_{4}$ and $\mathrm{LiCoPO}_{4}$ were found to have ferromagnetic (FM) ordering in our collinear calculations and the difference in ground state energies in FM and AFM ordering were found to be negligible. In the case of $\mathrm{Al}$ substitution, we have used FM ordering for all transition metal ions and collinear calculations. A correction for onsite electronic correlation at transition metal site is included through Hubbard U parameter, which is generally used for predicting accurately the electronic properties of compounds consisting of $d$ and $f$ block elements. Proper treatment of localization of $d$-orbital electrons leads to more accurate ordering of electronic states, bandgaps, magnetic moments, atomic positions and lattice parameters (Zhou et al 2004a). Cell parameters and atomic positions are relaxed to minimize the energy of each lithiated and de-lithiated structures. A grid-based Bader code developed by Henkelman's group is used (Henkelman et al 2006; Sanville et al 2007; Tang et al 2009) in estimating Bader charges of atoms in the compounds. In this method, a unit cell is partitioned into atomic volumes, such that the flux of electron density gradient is zero at every point on the interatomic surfaces. Orbital projections are done within this atomic volume instead of conventionally used spherical volumes (Sanville et al 2007). Bader's partitioning of charge density lets us to consider, most of the volume of the unit cell leading to better accuracy (Bader 1990) than spherical projection. Charge transfer maps, which show allocation of lithium valence electron to different atoms are prepared by subtracting de-lithiated compound electron density from the lithiated one. All the structures and charge transfer maps were created using Visual Molecular Dynamics (VMD) software (Humphrey et al 1999).

\section{Results and discussion}

$\mathrm{LiCoO}_{2}$ and $\mathrm{LiAlO}_{2}$ belong to $R \overline{3} m$ space group (figure 1(a)) and are hexagonal layered structures, whereas $\mathrm{LiMPO}_{4}(\mathrm{M}$ $=\mathrm{Fe}, \mathrm{Co}$ ) belongs to Pnma space group (figure 1(b)) and is an orthorhombic olivine structure. In hexagonal layered structure, lithium and metal occupy alternate layers with oxygen layers separating them. In olivine structure, the transition metal occupies octahedral site, phosphorous occupies tetrahedral site with oxygen atoms shared at the corners of octahedrons and tetrahedrons. Table 1 shows DFT calculated lattice parameters. These are marginally higher than experimental values, as expected for GGA approximation. We
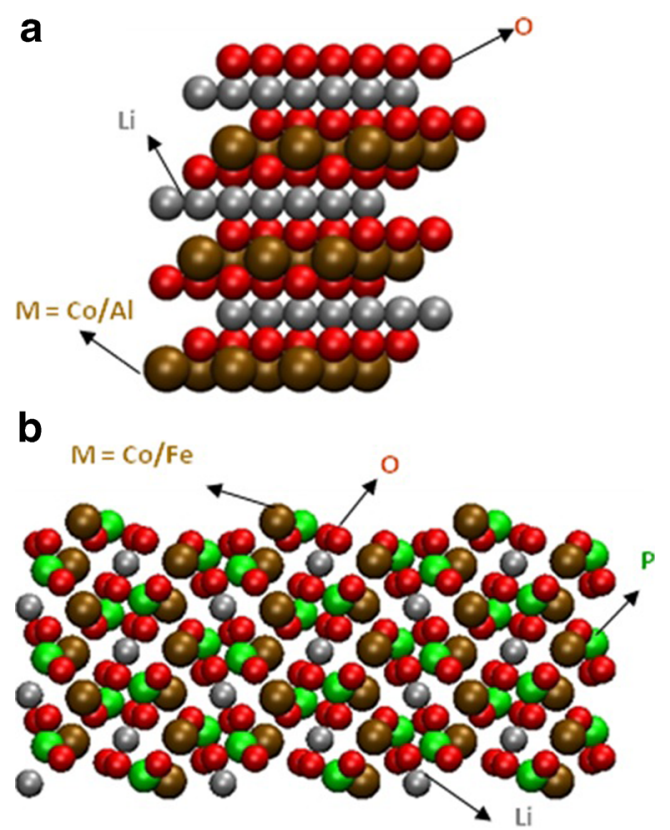

Figure 1. Crystal structure of cathode materials (a) layered $\mathrm{LiCoO}_{2}$ and (b) orthorhombic $\mathrm{LiFePO}_{4}$. 
Table 1. Lattice parameters of selected cathode materials.

\begin{tabular}{|c|c|c|c|c|c|c|}
\hline & \multicolumn{3}{|c|}{ From DFT $(\AA)$} & \multicolumn{3}{|c|}{ Expt $(\AA)$} \\
\hline & $\mathrm{a}$ & $\mathrm{b}$ & $\mathrm{c}$ & $a$ & $\mathrm{~b}$ & $\mathrm{c}$ \\
\hline $\mathrm{LiCoO}_{2}$ & $2 \cdot 84$ & $2 \cdot 84$ & $14 \cdot 1$ & $2 \cdot 82$ & $2 \cdot 82$ & $14 \cdot 04$ \\
\hline $\mathrm{CoO}_{2}$ & $2 \cdot 83$ & $2 \cdot 83$ & 15.99 & $\mathrm{NA}^{*}$ & $\mathrm{NA}^{*}$ & $\mathrm{NA}^{*}$ \\
\hline $\mathrm{LiAlO}_{2}$ & $2 \cdot 83$ & $2 \cdot 83$ & $14 \cdot 34$ & $2 \cdot 80$ & $2 \cdot 80$ & $14 \cdot 21$ \\
\hline $\mathrm{AlO}_{2}$ & $2 \cdot 89$ & $2 \cdot 89$ & $12 \cdot 06$ & $\mathrm{NA}^{*}$ & $\mathrm{NA}^{*}$ & $\mathrm{NA}^{*}$ \\
\hline $\mathrm{LiFePO}_{4}$ & $10 \cdot 41$ & $6 \cdot 06$ & $4 \cdot 75$ & $10 \cdot 33$ & $6 \cdot 01$ & $4 \cdot 69$ \\
\hline $\mathrm{FePO}_{4}$ & 9.97 & $5 \cdot 89$ & $4 \cdot 87$ & 9.82 & 5.79 & 4.79 \\
\hline $\mathrm{LiCoPO}_{4}$ & $10 \cdot 2$ & 5.92 & 4.7 & $10 \cdot 2$ & 5.92 & $4 \cdot 70$ \\
\hline $\mathrm{CoPO}_{4}$ & 9.66 & $5 \cdot 87$ & $4 \cdot 84$ & $10 \cdot 09$ & $5 \cdot 85$ & $4 \cdot 72$ \\
\hline
\end{tabular}

* Not applicable since fully delithiated layered structures do not exist in $R \overline{3} m$ space group.

Table 2. Electrochemical potential of cathode materials with aluminum substitution.

\begin{tabular}{ll}
\hline Cathode material & Calculated voltage in volts* \\
\hline $\mathrm{LiCoO}_{2}$ & 4.26 \\
$\mathrm{LiCo}_{0.67} \mathrm{Al}_{0.33} \mathrm{O}_{2}$ & $4.54(4.2)$ \\
$\mathrm{LiCo}_{0.33} \mathrm{Al}_{0.67} \mathrm{O}_{2}$ & $4.81(4.7)$ \\
$\mathrm{LiFePO}_{4}$ & $3.51(3.47)$ \\
$\mathrm{LiFe}_{0.75} \mathrm{Al}_{0.25} \mathrm{PO}_{4}$ & 2.64 \\
$\mathrm{LiFe}_{0.5} \mathrm{Al}_{0.5} \mathrm{PO}_{4}$ & 1.75 \\
$\mathrm{LiCoPO}_{4}$ & $4.62(4.73)$ \\
$\mathrm{LiCo}_{0.75} \mathrm{Al}_{0.25} \mathrm{PO}_{4}$ & 3.75 \\
$\mathrm{LiCo}_{0.5} \mathrm{Al}_{0.5} \mathrm{PO}_{4}$ & 2.78 \\
\hline
\end{tabular}

*Previous simulation results shown in parentheses (Ceder et al 1998; Zhou et al 2004a, b)

Table 3. Charge transfer analysis of unsubstituted/Al substituted cathode materials.

\begin{tabular}{|c|c|c|c|c|c|c|c|}
\hline \multirow[t]{3}{*}{ Cathode materials } & \multirow[t]{3}{*}{ Voltage (V) } & \multicolumn{6}{|c|}{ Fractional charge transfer per formula unit } \\
\hline & & \multirow[b]{2}{*}{ From Li } & \multicolumn{4}{|l|}{ Onto } & \multirow[b]{2}{*}{$\left(\mathrm{B}_{\mathrm{O}} / \mathrm{B}_{\mathrm{Li}}\right) * 100$} \\
\hline & & & $\mathrm{O}$ & TM & $\mathrm{Al}$ & $\mathrm{P}$ & \\
\hline $\mathrm{LiCoO}_{2}$ & $4 \cdot 26$ & $0 \cdot 865$ & 0.748 & $0 \cdot 117$ & 0 & - & $86 \cdot 37$ \\
\hline $\mathrm{LiCo}_{0.67} \mathrm{Al}_{0.33} \mathrm{O}_{2}$ & 4.54 & $0 \cdot 863$ & 0.789 & $0 \cdot 069$ & $0 \cdot 004$ & - & $91 \cdot 42$ \\
\hline $\mathrm{LiCo}_{0 \cdot 33} \mathrm{Al}_{0.67} \mathrm{O}_{2}$ & $4 \cdot 81$ & $0 \cdot 863$ & $0 \cdot 816$ & 0.039 & $0 \cdot 008$ & - & $94 \cdot 55$ \\
\hline $\mathrm{LiFePO}_{4}$ & $3 \cdot 51$ & $0 \cdot 878$ & $0 \cdot 446$ & $0 \cdot 412$ & 0 & $0 \cdot 020$ & $50 \cdot 80$ \\
\hline $\mathrm{LiFe}_{0.75} \mathrm{Al}_{0.25} \mathrm{PO}_{4}$ & $2 \cdot 64$ & $0 \cdot 877$ & $0 \cdot 393$ & 0.461 & $0 \cdot 001$ & $0 \cdot 021$ & $44 \cdot 84$ \\
\hline $\mathrm{LiFe}_{0.50} \mathrm{Al}_{0.50} \mathrm{PO}_{4}$ & 1.75 & 0.879 & $0 \cdot 328$ & $0 \cdot 514$ & 0.003 & 0.033 & $37 \cdot 32$ \\
\hline $\mathrm{LiCoPO}_{4}$ & $4 \cdot 62$ & 0.874 & 0.548 & $0 \cdot 315$ & 0 & $0 \cdot 010$ & $62 \cdot 70$ \\
\hline $\mathrm{LiCo}_{0.75} \mathrm{Al}_{0.25} \mathrm{PO}_{4}$ & 3.75 & $0 \cdot 875$ & $0 \cdot 482$ & $0 \cdot 391$ & 0.001 & $0 \cdot 016$ & $55 \cdot 08$ \\
\hline $\mathrm{LiCo}_{0.50} \mathrm{Al}_{0.50} \mathrm{PO}_{4}$ & 2.78 & 0.877 & $0 \cdot 389$ & 0.477 & 0.002 & 0.026 & $42 \cdot 37$ \\
\hline
\end{tabular}

determine the average equilibrium voltage over a full cycle $\left(V_{\text {cell }}\right)$ of a battery from Nernst equation:

$$
V_{\text {cell }}=-\Delta G / z F
$$

where $z$ is the charge on lithium ion in the electrode $(z=1), F$ the Faraday constant and $\Delta G$ the free energy change in the overall reaction. Volume and entropic effects are neglected, so that $\Delta G$ can be approximated by the change in ground state internal energy (Ceder et al 1997).

Earlier experimental and theoretical studies indicate that aluminum substitution in $\mathrm{LiCoO}_{2}$ increases the battery potential considerably (Ceder et al 1998). Here, we calculate electrochemical potential for $\mathrm{LiFePO}_{4}, \mathrm{LiCoPO}_{4}$ and 
$\mathrm{LiCoO}_{2}$ with different amounts of aluminum substituting transition metal (table 2). The trend of electrochemical potential at different levels of $\mathrm{Al}$ substitution in $\mathrm{LiCoO}_{2}$ in our work agrees qualitatively with previous work done by Ceder et al (1998) mentioned above. However, the quantitative difference in values between our and Ceder's work arises from difference in the methodology adopted. While Ceder et al adopted local density approximation (LDA) for exchange-correlation functional, we have used generalized gradient approximation $(\mathrm{GGA}+\mathrm{U})$. Of the possible configurations in substituted compounds, the ones with minimum ground state energies were considered to evaluate electrochemical potentials and other properties presented here. It is seen that $\mathrm{Al}$ substitution behaves differently in oxides and phosphates. As one can see from table 2, electrochemical potential increases with $\mathrm{Al}$ substitution in oxides, whereas it decreases in phosphates.

To understand the opposite effects of $\mathrm{Al}$ substitution on electrochemical potential of $\mathrm{LiCoO}_{2}$ and $\mathrm{Li}(\mathrm{Fe} / \mathrm{Co}) \mathrm{PO}_{4}$, we carried out Bader charge analysis (Bader 1990), The results are shown in table 3. This is an intuitive method for visualizing atoms in the molecules. The charge enclosed within the Bader volume is a good approximation to the total electronic charge of an atom. For lithiated and de-lithiated compounds, we calculated the charges enclosed in Bader volumes and thus, we determined the charge of lithium that is transferred from lithiated state to de-lithiated state. It is seen that extra charge transferred onto oxygen atoms $\left(B_{\mathrm{O}}\right)$ due to substitution effect correlates with increased voltage (figure 2 ). In hexagonal-layered cobalt oxide, large fraction of charge gets transferred to oxygen with increased aluminum substitution. In contrast, transition metal shows significant participation in charge transfer process with increased $\mathrm{Al}$ substitution in olivine phosphates (figure 3). From figures $3(a-c)$, we can see substantial increase in charge distribution around oxygen compared to that of cobalt with increased aluminum substitution. In contrast, for olivine phosphates, figures 3(d) and (e) show increase in charge distribution around iron compared to oxygen with increased aluminum substitution. The opposing tendencies observed in layered oxides and olivine phosphates indicate the role of structure and chemical bonding in these materials. In substituted $\mathrm{LiCoO}_{2}$, inability of aluminum to take up electron forces (due to lack of $d$-orbitals) increased participation of oxygen in the electron exchange. It has been speculated that excess electron on the electronegative oxygen atom rather than on transition metal lowers electrostatic energy and results in higher voltage (Ceder et al 1998). However, the underlying chemical phenomena need to be well understood. This will also help us to explain opposite effects in $\mathrm{Al}$ substituted phosphates, where lower charge transfer to oxygen leads to reduced voltage.

In order to understand the underlying chemistry, we carry out total and projected density of states (DOS) calculations in Bader volumes with modified VASP code using a module developed by Henkelman et al (2006), that projects DOS in

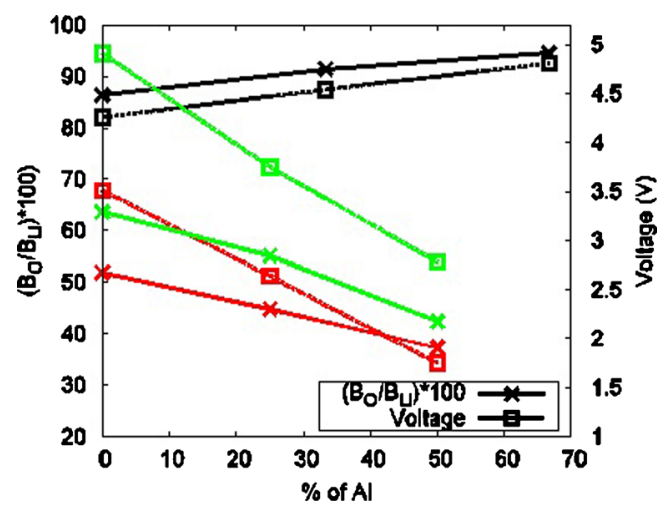

Figure 2. Effect of aluminum substitution on charge transfer to oxygen from lithium and on voltage. Black lines are for $\mathrm{LiCoO}_{2}$, red lines for $\mathrm{LiFePO}_{4}$ and green lines for $\mathrm{LiCoPO}_{4}$.

Bader volumes (density of states projections in Bader volume). DOS near Fermi energy shows sharp features associated with $d$-orbital bands of cobalt in $\mathrm{LiCoO}_{2}$ and relatively broader $d$-orbital bands of $\mathrm{Fe}(\mathrm{Co})$ in $\mathrm{LiFePO}_{4}\left(\mathrm{LiCoPO}_{4}\right)$. Oxygen states are separated from $d$ states and are deeper in energy in $\mathrm{LiCoO}_{2}$. DOS of phosphates show wider bands and there is no separation between oxygen $p$-orbital and TM $d$-orbital. A strong hybridization between oxygen $p$-orbital and transition metal $d$-orbital is observed (figure 4). This shows the stronger covalency in TM-O bond for phosphates compared to that of oxides. So charge transfer from lithium to oxygen is less in the case of phosphates compared to oxides which is evident from table 3 .

Aluminum substitution in phosphates forces more charge onto transition metal and phosphorus, thereby decreasing the charge transfer to oxygen. This increases the electrostatic energy of $\mathrm{LiFePO}_{4}\left(\mathrm{LiCoPO}_{4}\right)$ system and decreases the battery potential. This is consistent with the fact that phosphate ion slows down the oxygen reduction. The presence of phosphorus in these compounds leads to increased covalency in $\mathrm{TM}-\mathrm{O}$ bond thereby leading to decreased substitution related charge transfer to oxygen.

We calculated the average energy levels of individual orbitals and electron occupancies of all atoms in the compound from projected density of states. This was done by integrating the partial density of states of specific orbitals of atoms upto fermi level. The average energy level of specific orbital of atoms was also calculated in the same way. Figure 5 shows energy levels of individual orbitals on $y$-axis. The occupancy values of specific orbitals are shown along $x$-axis. The plots for $\mathrm{LiCoO}_{2}, \mathrm{LiAl}_{0.33} \mathrm{Co}_{0.67} \mathrm{O}_{2}, \mathrm{LiFePO}_{4}$ and $\mathrm{LiFe}_{0.75} \mathrm{Al}_{0.25} \mathrm{PO}_{4}$ are shown. In case of $\mathrm{LiFePO}_{4}$ and $\mathrm{LiFe}_{0.75} \mathrm{Al}_{0.25} \mathrm{PO}_{4}$, the spin-down occupancies are shown on negative side of $x$-axis. From figures 5 (a) and (b), it can be seen that aluminum substitution increases the separation between oxygen $p$-orbital energy levels and that of cobalt 

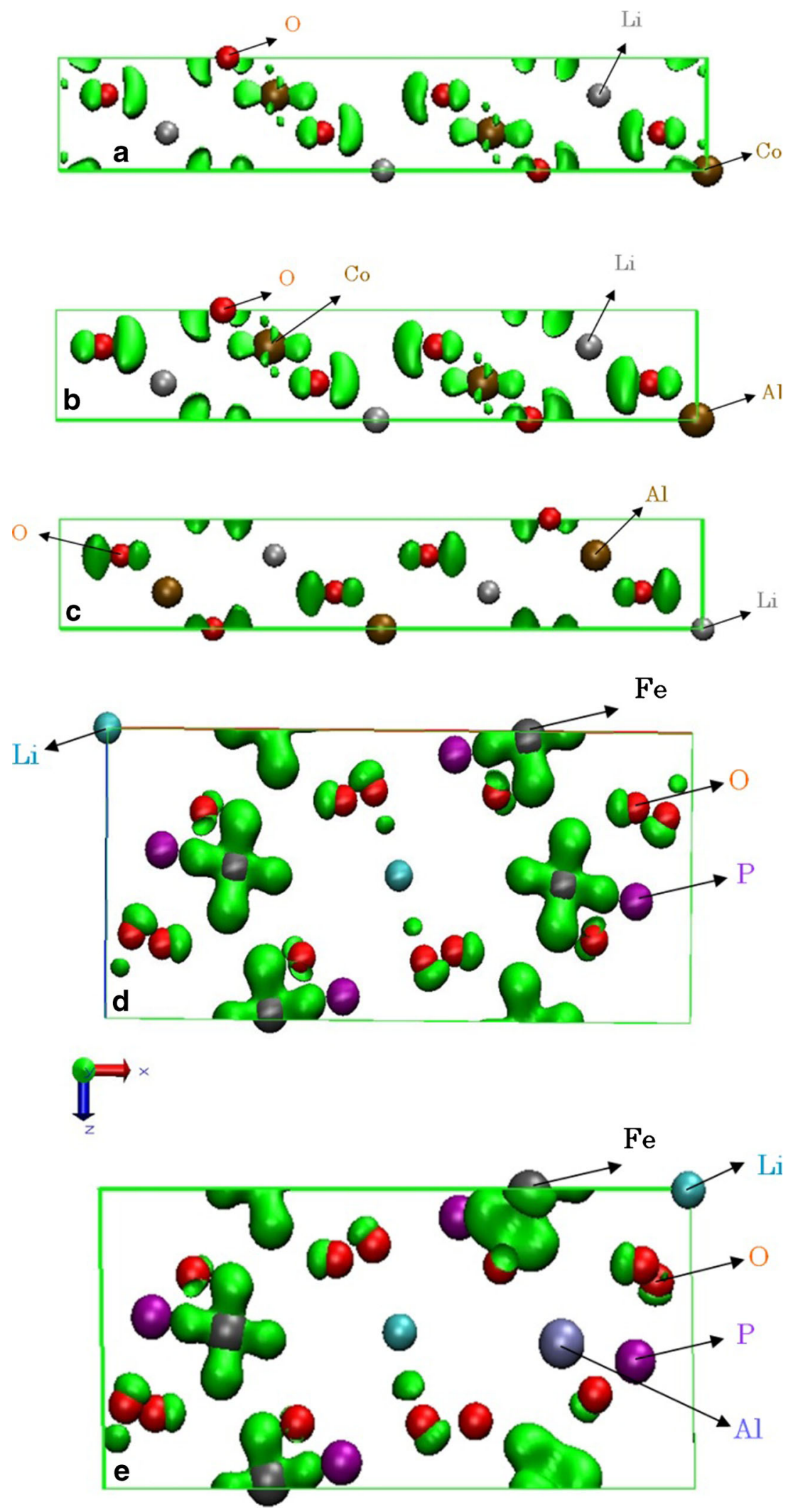

Figure 3. Charge transfer map showing the contrast between substitution effect in oxide and phosphate material (a) $\mathrm{LiCoO}_{2}$ (b) $\mathrm{LiAl}_{0.33} \mathrm{Co}_{0.67} \mathrm{O}_{2}$ (c) $\mathrm{LiAlO}_{2}$ (d) $\mathrm{LiFePO}_{4}$ (e) $\mathrm{LiFe}_{0.75} \mathrm{Al}_{0.25} \mathrm{PO}_{4}$. 


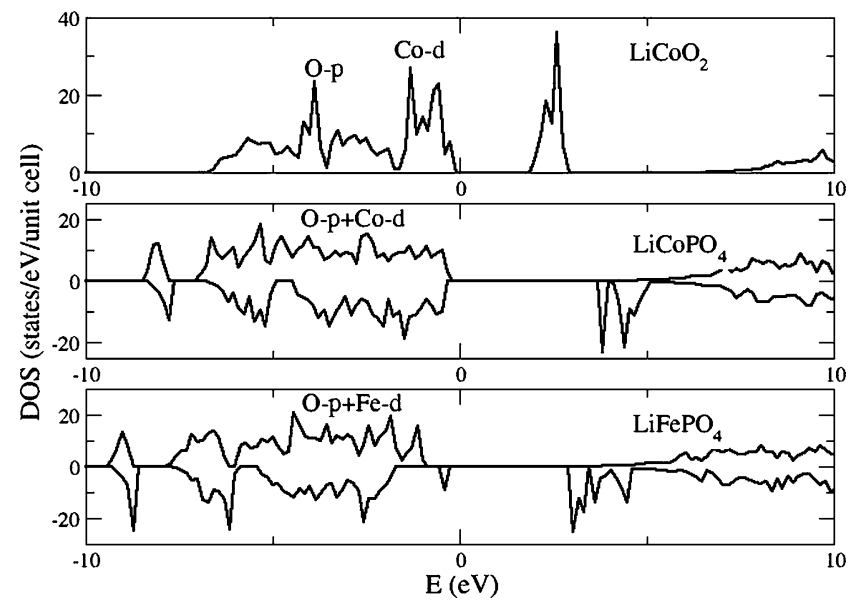

Figure 4. Total density of states for $\mathrm{LiCoO}_{2}, \mathrm{LiCoPO}_{4}$ and $\mathrm{LiFePO}_{4}$. Origin has been shifted to Fermi energy level.
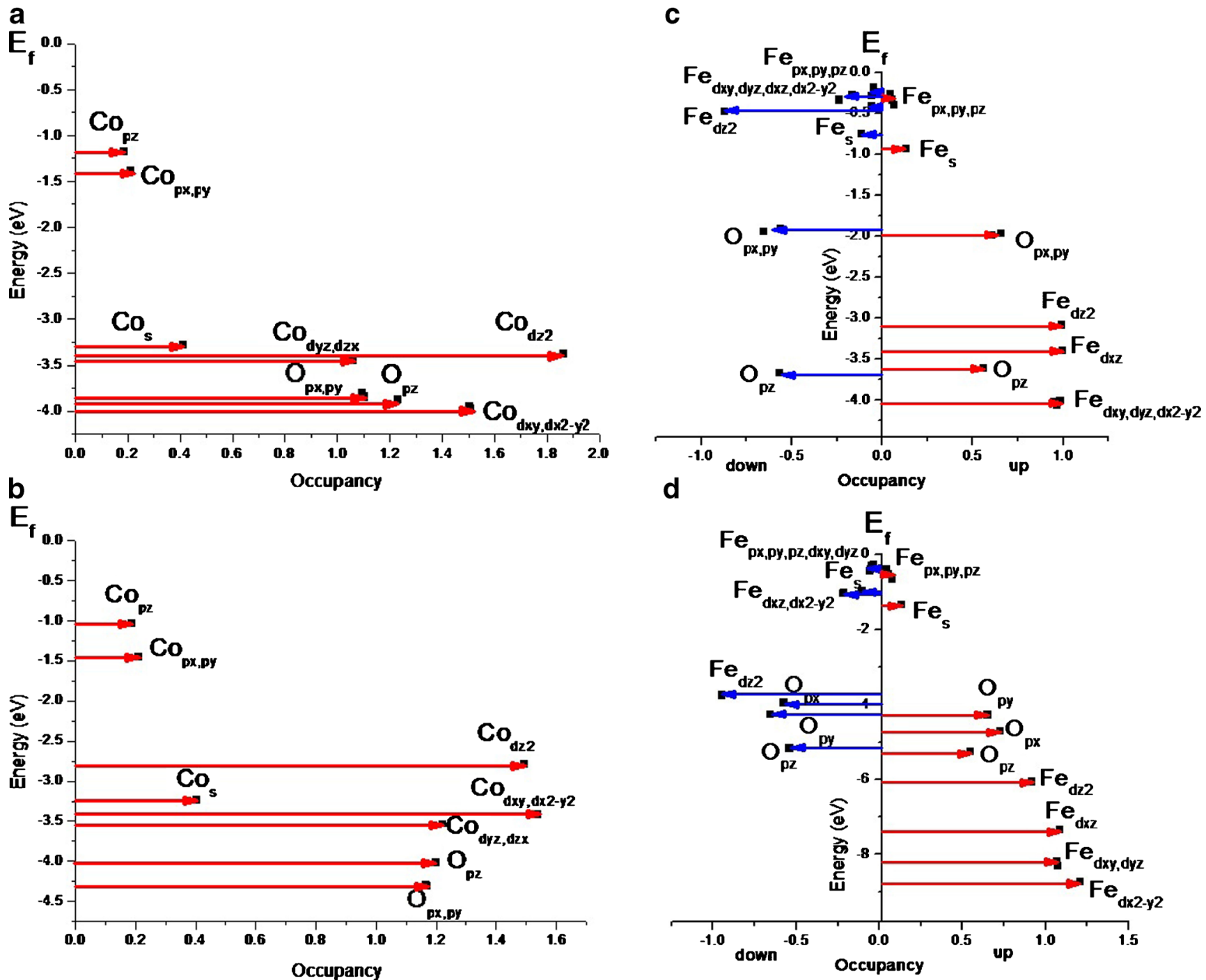

d

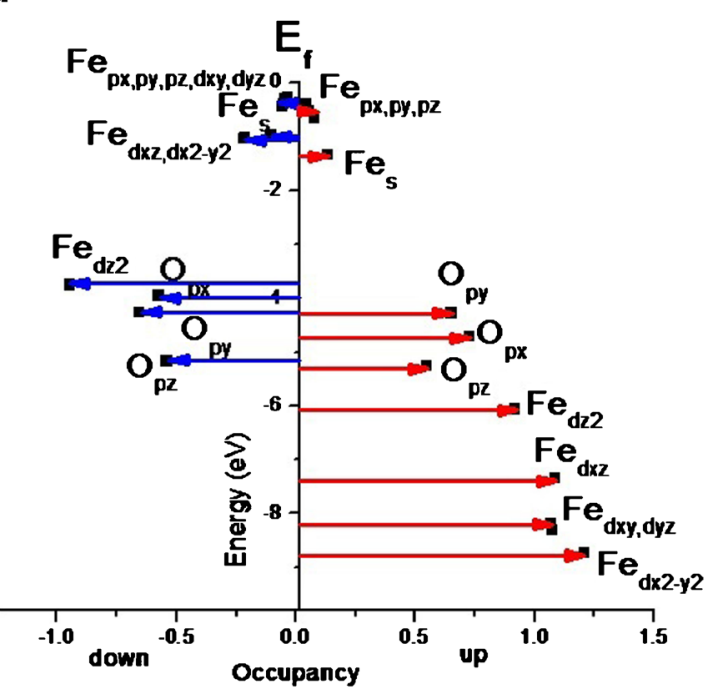

Figure 5. Energy level and occupation diagrams for TM and Oxygen for (a) $\mathrm{LiCoO}_{2},(\mathbf{b}) \mathrm{LiAl}_{0 \cdot 33} \mathrm{Co}_{0 \cdot 67} \mathrm{O}_{2},(\mathbf{c}) \mathrm{LiFePO}_{4}$ and (d) $\mathrm{LiFe}_{0.75} \mathrm{Al}_{0.25} \mathrm{PO}_{4}$. The spin-up and spin-down occupancies are shown along the positive and negative $x$-axes, respectively. 
Table 4. Position of oxygen $p$-orbital with respect to transition metal $d$-orbital.

\begin{tabular}{ll}
\hline Cathode materials & $\begin{array}{l}\text { Shift in the position } \\
\text { of O- } p \text { orbital } \\
\text { w.r.t. TM- } d \\
\text { orbital }(\mathrm{eV})\end{array}$ \\
\hline $\mathrm{LiCoO}_{2}$ & -0.195 \\
$\mathrm{LiCo}_{0.67} \mathrm{Al}_{0 \cdot 33} \mathrm{O}_{2}$ & -0.928 \\
$\mathrm{LiCoPO}_{4}$ & $1 \cdot 176$ \\
$\mathrm{LiCo}_{0.75} \mathrm{Al}_{0.25} \mathrm{PO}_{4}$ & 1.904 \\
$\mathrm{LiFePO}_{4}$ & 0.498 \\
$\mathrm{LiFe}_{0.75} \mathrm{Al}_{0 \cdot 25} \mathrm{PO}_{4}$ & $2 \cdot 111$ \\
\hline
\end{tabular}

$d$-orbital energy levels. However, in case of aluminum substitution in $\mathrm{LiFePO}_{4}$, the above separation between oxygen $p$-orbitals and transition metal $d$-orbitals is less. This indicates increased covalency with substitution of aluminum in phosphates. The relative shift of the oxygen $p$ orbitals with respect to transititon metal $d$-orbitals are shown in table 4 . It is evident from the table that relative positions of oxygen $p$-orbitals and transition metal $d$-orbitals play a role in determining the electrochemical potential of the cathode material.

\section{Conclusions}

In agreement with Ceder et al (1998), aluminum substitution was found to enhance the electrochemical potential in $\mathrm{LiCoO}_{2}$. To correlate this finding with chemical and structural aspects, we did a detailed study of charge transfer and energy levels due to substitution.

Bader charge analysis indicates that the valence charge of lithiumis distributed more on oxygen when some cobalt is replaced with aluminum in $\mathrm{LiCoO}_{2}$. However, this is not applicable to all cathode materials. Comparative study of aluminum substitution in olivine phosphates shows why battery potential gets lowered. We explain this quantitatively by Bader charge analysis and show that lower fraction of lithium valence charge is distributed onto oxygen atoms in phosphates on aluminum substitution. Orbital energy diagrams show the relative position of oxygen $p$-states with respect to the transition metal $d$-states in the compounds. With the substitution of aluminum, we could see that, the energy levels of oxygen $p$-states get lowered compared to transition metal $d$-states in oxides whereas, the reverse happens in the phosphates. Positioning of oxygen $p$-orbital energy levels relative to TM $d$ orbital energy levels determines the electrochemical potentials and explains the contrasting trends observed in the oxides and phosphates studied here.
A detailed analysis with respect to ligand field theory would give more insights into the quantitative results for different battery materials.

\section{Acknowledgments}

We would like to express our gratitude to $\mathrm{R}$ Krishnan, V S Arunachalam (CSTEP) and A K Shukla (SSCU, IISc) for their advice during this study. We would like to thank V S Mahalingam, Director, Centre for Artificial Intelligence and Robotics, Bangalore, for providing the computational facilities for carrying out the simulations. The assistance provided by Tanmay Sarkar (CSTEP) in preparation of the manuscript is sincerely acknowledged. This work was funded by Defense Research and Development Organization, India, via grant no. ERIP/ER/0906002/M/01/1201.

\section{References}

Bader R F W 1990 Atoms in molecules: A quantum theory (New York: Oxford University Press)

Blochl P E 1994 Phys. Rev B50 17953

Ceder G, Aydinol M K and Kohan A F 1997 Comput. Mater. Sci 8 161

Ceder G, Chiang Y M, Sadoway D R, Aydiniol M K, Jang Y I and Huang B 1998 Nature 392694

Ebner W, Fouchard D and Xie L 1994 Solid State Ionics 69238

Henkelman G, Arnaldsson A and Jonsson H 2006 Comput. Mater. Sci. 36254

Humphrey W, Dalke A and Schulten K 1999 J. Mol. Graphics 14 33

Kang K, Meng Y S, Breger J, Grey C P and Ceder G 2006 Science 311977

Kresse G and Joubert D 1999 Phys. Rev. B59 1758

Ong S P, Wang L, Kong B and Ceder G 2008 Chem. Mater. 201798

Padhi A K, Nanjundaswamy K S and Goodenough J B 1997 J. Electrochem. Soc. 1441188

Perdew J P, Chevary J A, Vosko S H, Jackson K A, Pederson M R, Singh D J and Fiolhais C 1992 Phys. Rev. B46 6671

Sanville E, Kenny S D, Smith R and Henkelman G 2007 J. Comp. Chem. 28899

Sathiya M, Hemalatha K, Ramesha K, Shukla A K and Prakash A S 2011 Bull. Mater. Sci. 341693

Shalini R, Munichandraiah N and Shukla A K 2001 J. Power Sources $\mathbf{1 0 2} 322$

Tang W, Sanville E and Henkelman G 2009 J. Phys: Condens. Matter 21084204

Wang Li, Zhang Liwu, Li Jianjun, Gao Jian, Jiang Changyin and He Xiangming 2012 Int. J. Electrochem. Sci. 73362

Zhou F, Cococcioni M, Kang K and Ceder G 2004a Electrochem. Commun. 61144

Zhou F, Kang K, Maxisch T, Ceder G and Morgan D 2004b Solid State Commun. 132181 